\title{
13
}

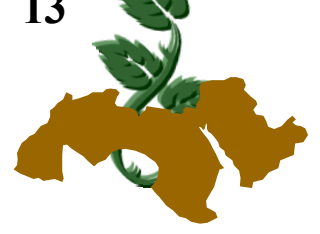

مجلة اتحاد الجامعات العربية

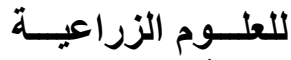

جامعة عين شمس ، القبس ، القاهرة

مجلد(19)، عدد (1)، 23-13، 2011

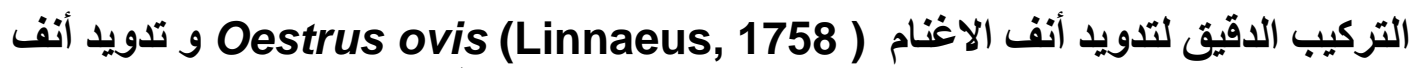
الجمال Cephalopina titillator (Clark 1797 بواسطة المجهر الاكتروني الماسح (SEM)

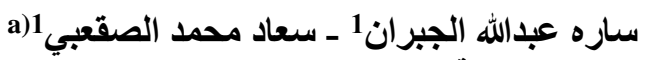

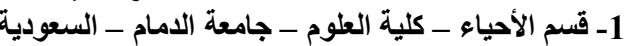

(عنوان المراسلة : المملكة العربية السعودية صاعب ب 838 الرمز البريدي 31113 جامعة الامام بالدمام)

a) E-mil: $\underline{\text { dr-alsaqabi@hotmail.com }}$

التي تمت بالمملكة العربيـة السعوديه في منـاطق نربيـة

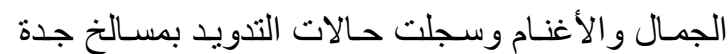

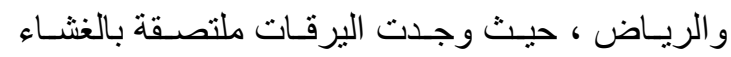

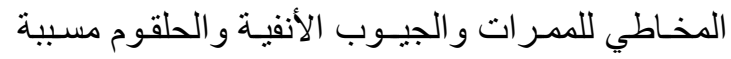

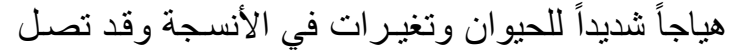

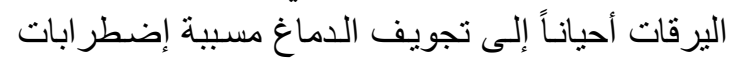
عصبية قد تفضي إلى الموت. وتحدث اليرقات الئات أيضاً

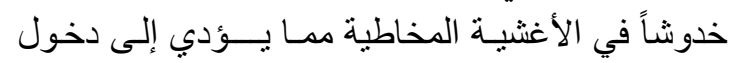

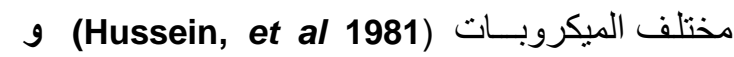
هذا النـوع شـائع الوجـود في منساطق تربيـة الجمـال

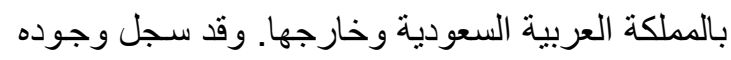

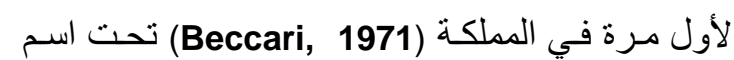
و وأعـاد تسـجيل وجـوده مـن Cephalopina titillator (Buttiker and Zumpet, مسلخ بالرياض كل من

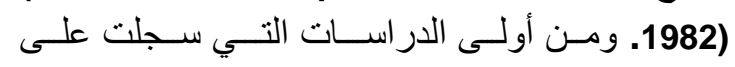
التدويد في أنف الأغنام في المملكة العربيـة السعودية

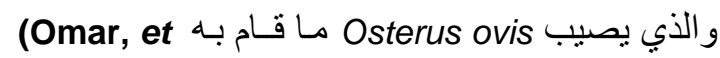
(Grammer, et al 1995; كما قام كل من al 1988) بوصف الطـور اليرقي Stevens, et al 1991)

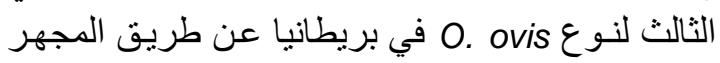

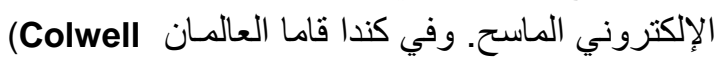
بدر اسـة التر اكيب الجليديـة Gastrophilus الحسبة المتو اجدة على جسم برقات

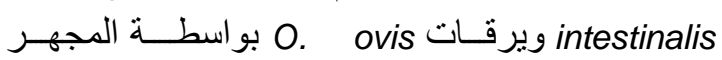
الإلكتروني الماستح. بينما درس
الكلمــات الدالــة: تدويـــ ، SEM ، المملكــة العربيـة

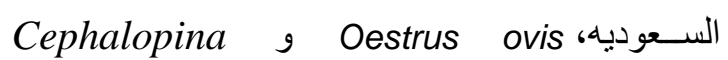
titillator

\section{ABSTRACT الموجز}

أظهرت الدراسة التركيب الدقيق لنو عين من برقات

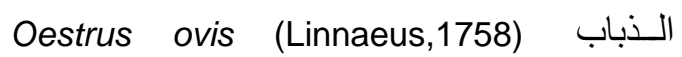
Cephalopina titillator (Clark 1797),

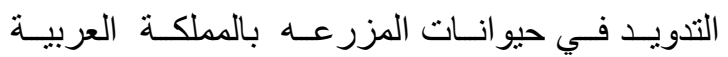

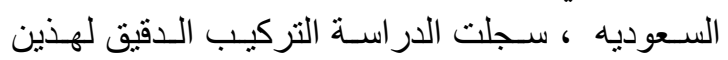

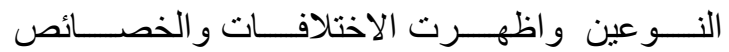

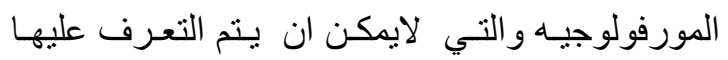

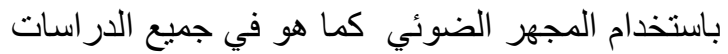

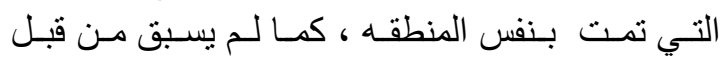

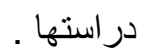

\section{INTRODUCTION المقدمة}

يعتبر التدويد Myasis و على الأخص نغف الأنف

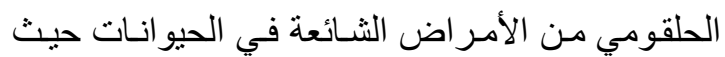

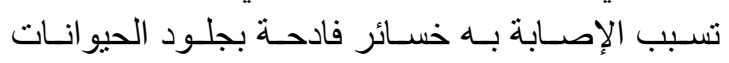

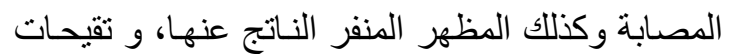

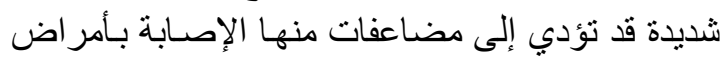
أخرى. وذكر (دبور وموسى 1981) بعض الد الدراسـات

(سلم البحث فى 29 سبتمبر 2010) (وفى ) (ووفث على البحث فى 17 يناير 2011) 


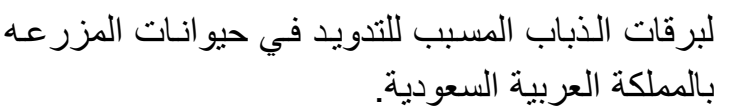

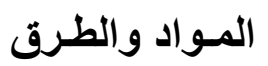

\section{MATERIALS AND METHODS}

أُجريت هذه الدراسة لتصنيف ووصف لنو عين من

يرقات الذباب (Linnaeus,1758) Cephalopina titillator (Clark, 1797) حيوانـات المزر عـة بالمنطقة الثرقية بالمملكة العربيـة

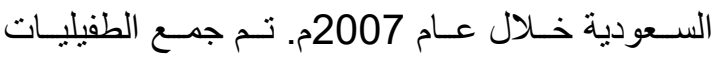

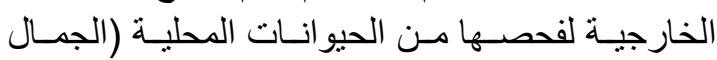
Camelus dromedarius

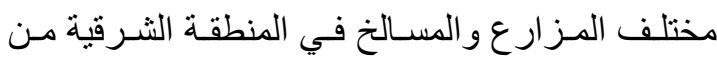

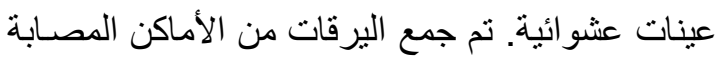

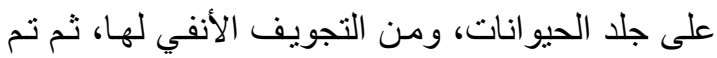

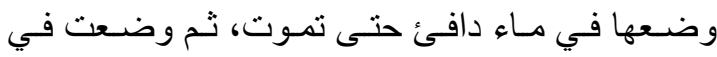

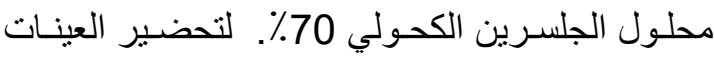

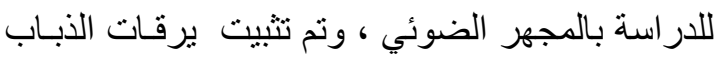

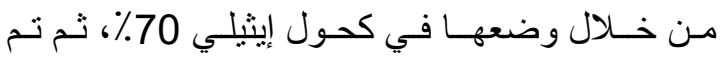

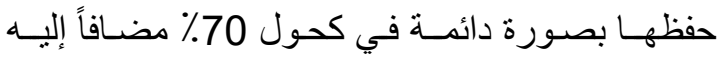
جلســرين 5\%. وتـم عمـــل شـر ائح دائمسـة للعينـات باسـتخدام طريقة (Pritchard \& Kruse, 1982)

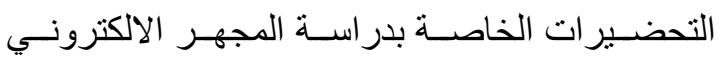

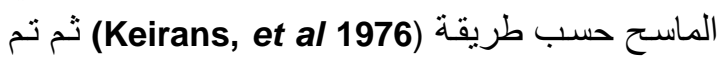

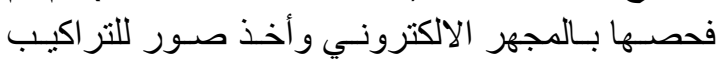

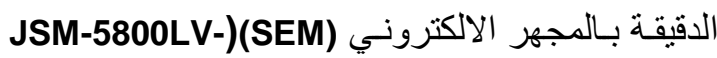

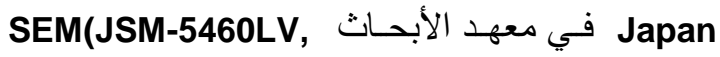

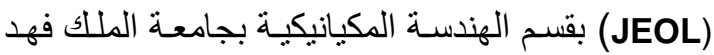

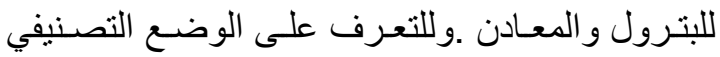

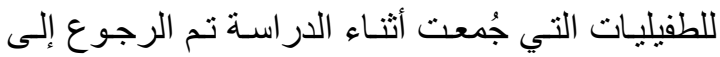

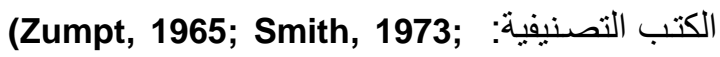
Bland and Jaques, 1980; Borror, et al

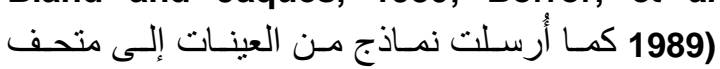

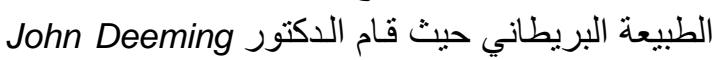
بتأكيد التعريف.
، O. ovis 1997 في بريطانيا التر اكيب الدقيقة لجليد التيدة

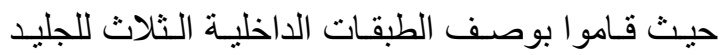

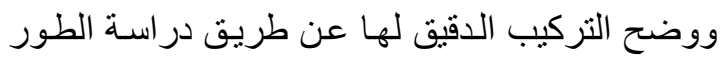
اليرقي الثالث.وقد قام (Giannetto, et al 1999)

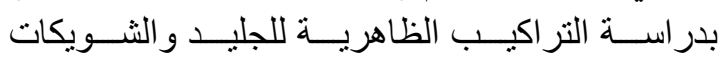

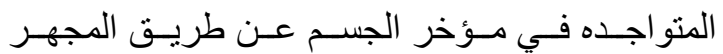

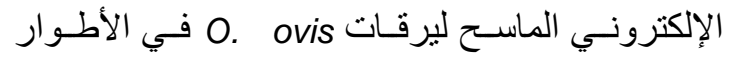

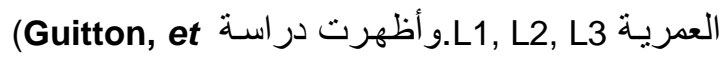
التركيب الـقيق ليرقات الأطـوار الثلاثـة al 2001)

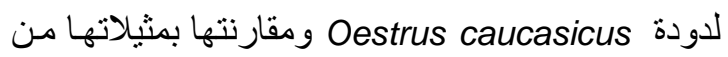
برقات Oestrus ovis. ومن الدر اسـات التي تمت في مني

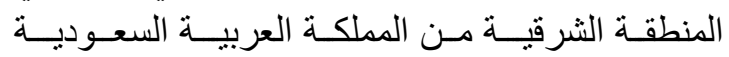

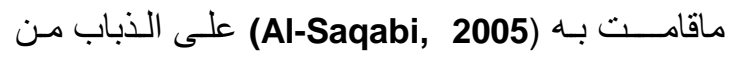

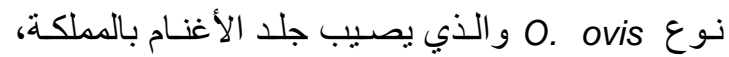

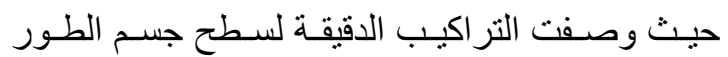

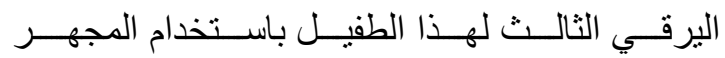

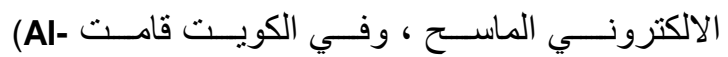
O. ovis بدر اسـة يرقات التي Behbehani, 2006)

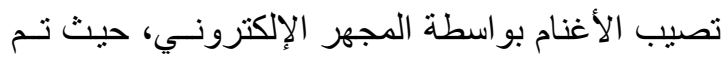

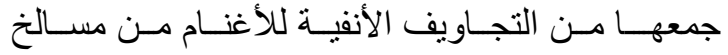
الشويخ. وفي مصر قام (Zayed et al 2008) بدر اسـة

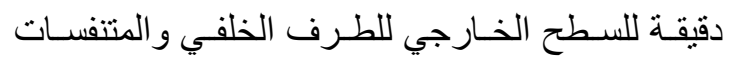

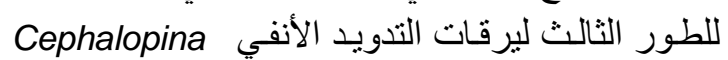
titillator, Oestrus ovis and Rhinoestrus purpureus و التـي تصيب الجمال و الأغنام و الحمير ،

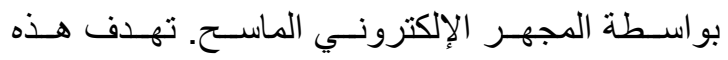

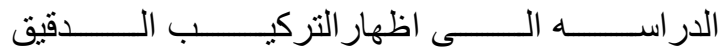

\section{النتائج والمناقشة}

Arab Univ. J. Agric. Sci., 19(1), 2011 
الجسم ملمساً خشناً، تظهر هذه الأشو الك بشكل واضح

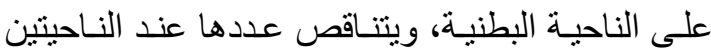
الجانبيتين، كما ينعدم تو اجدها في الناحية الظهرية .

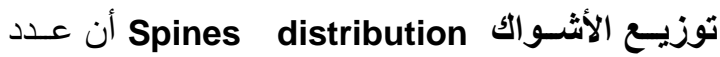

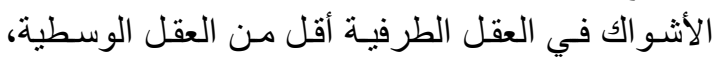

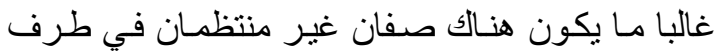

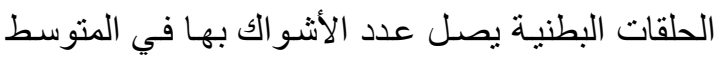
(30 شوكة) ، بينما تحتوي الحلقات الوسطية على (3-

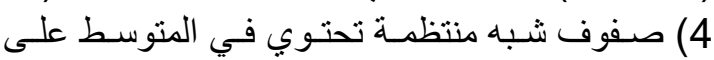
. (65) شوكة)

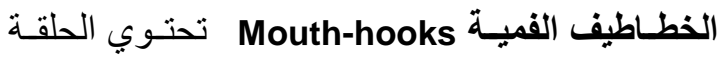

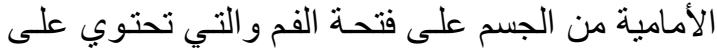

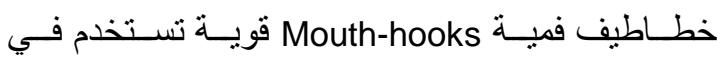

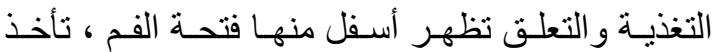
الخطاطيف اللون الأسود وتظهر مقدمتها بشكل بـارز

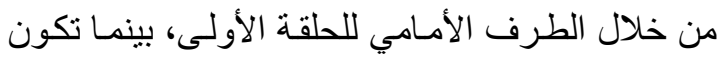

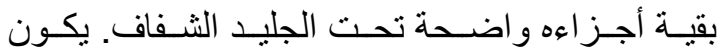

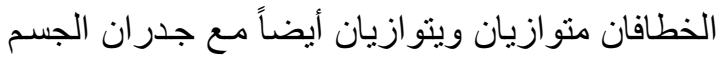
الجانبية حيث يتخذان وضع عمودي مع حلقات الجسم.

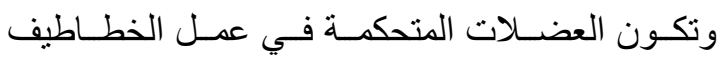
واضحة جداً في الفحص من خلال المجهر الضونئي .

الثغور التنفسية Spiracles تحتوي الحلقة الأخيرة من

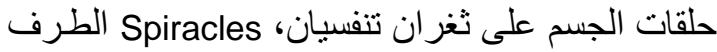

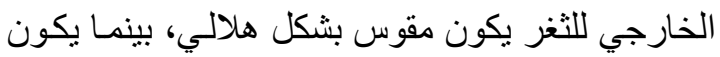

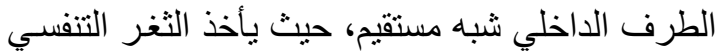

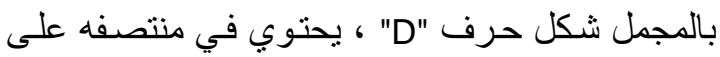

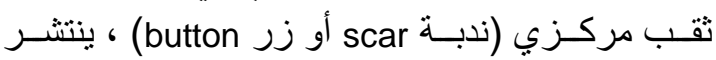

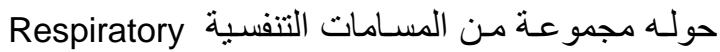
pore الدقيقة و التي تترتب على التى شكل أشعــة مركز هـا الثقب المركزي لتحتل أغلب مسـاحة القرص التبكي التفسي Spiracular disc حيث أن مستوى تو اجدها في وسط الحلقة ينخفض عن فئن

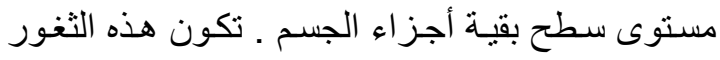
بنية اللون، ويندرج اللون إلى البني الداكن كلمـا كبرت

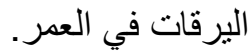

\section{RESULTS AND DISCUSSION}

أظهرت الدر اسة نو اجد إصـابة بالتدويد الأنفي في والإل الأغنام و الجمال، وفي جلد الجمال و الأغنام في مختلف التالف الأفي

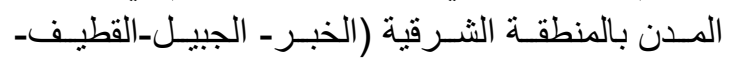
الأحساء-عنك-الدمام). كما بينت الدر اسة تو اجد الطور

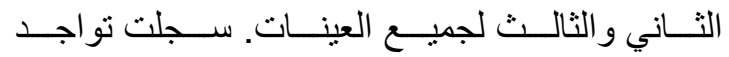
Cephalopina titillator (Clark, 1797)

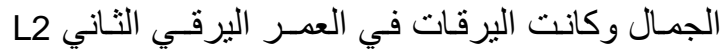
و الثالث L3 و و Oesterus ovis (Linnaeus, 1758) سجلت تواجدها في أنف الأغنـام في الطور اليرقي

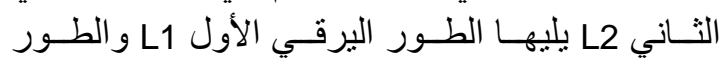

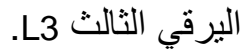

\section{تلدويد أنف الغتم Oesterus ovis}

\section{الوصف Description : (أشكال رقم 1 و 2 و3)}

apodous اليرقـات دوديـة الثكل عديمـة الأرجل

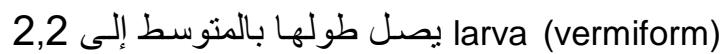

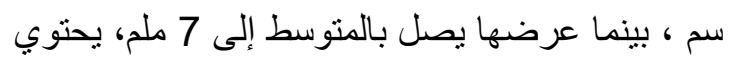

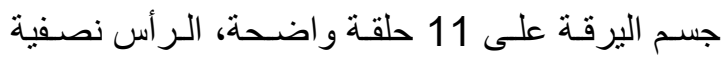

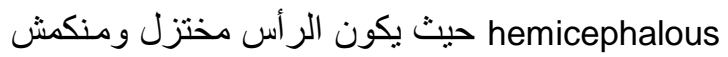
داخل الصدر، تكون الحلقة الأولى منـه أطول من بقيَة

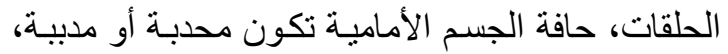

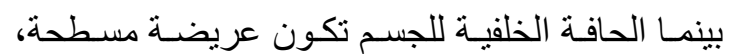

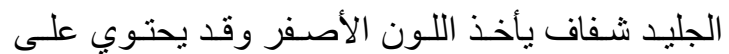

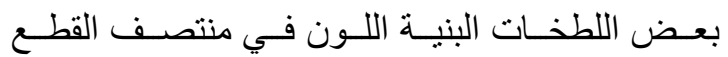

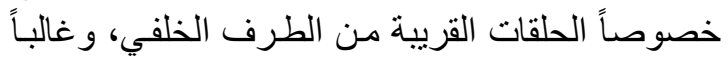

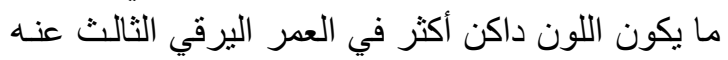

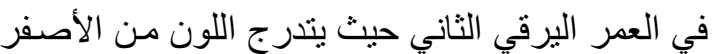

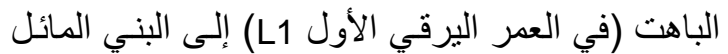

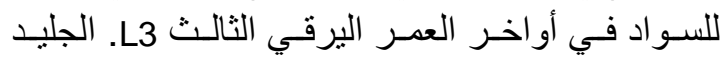
integument

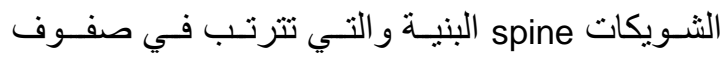
(تكون موزعـة في صفوف rows من 2-4) على كل حلقة segment من حلقات السطح البطني و التي تعطي 
(द)

(ب)

(1)

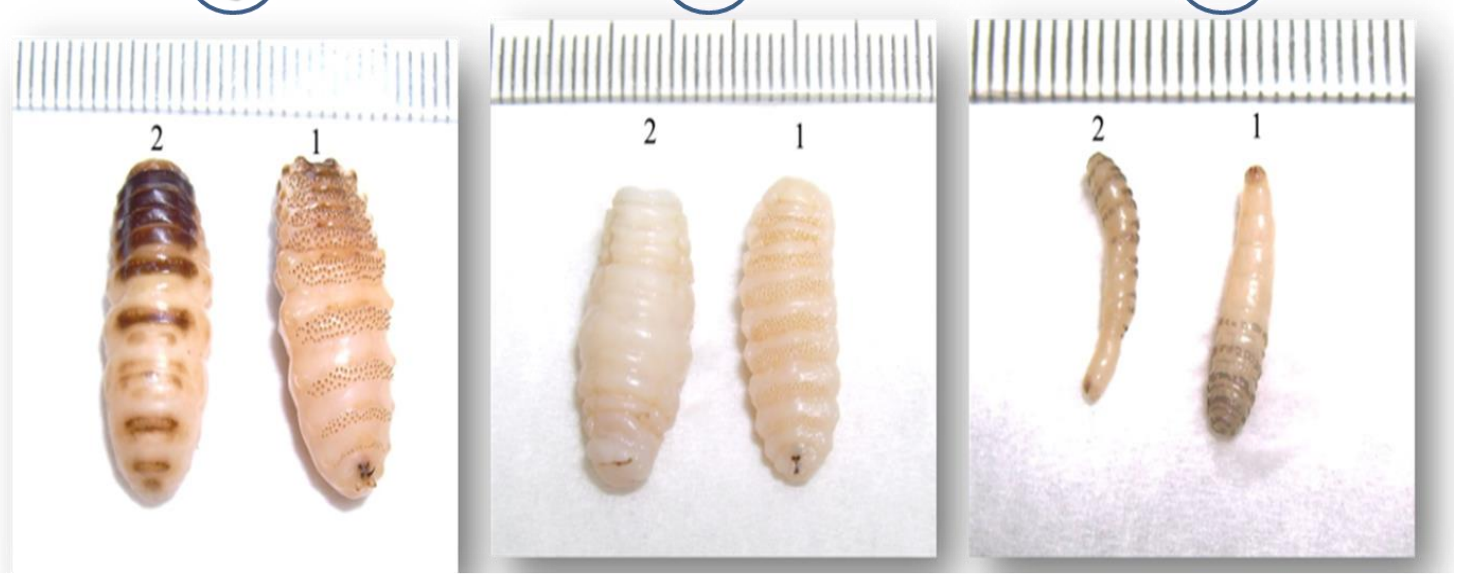

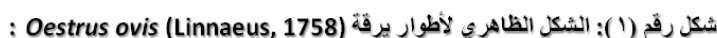
أ - الشكل الظاهري الليرقة في العمر الأول. ب - الثكل الظاهري الليرقة في العمر الثاني. ج - الشكل الظاهري لليرقة في العمر الثاثث. ا - منظر بطني.

(द)

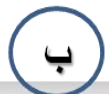

(i)
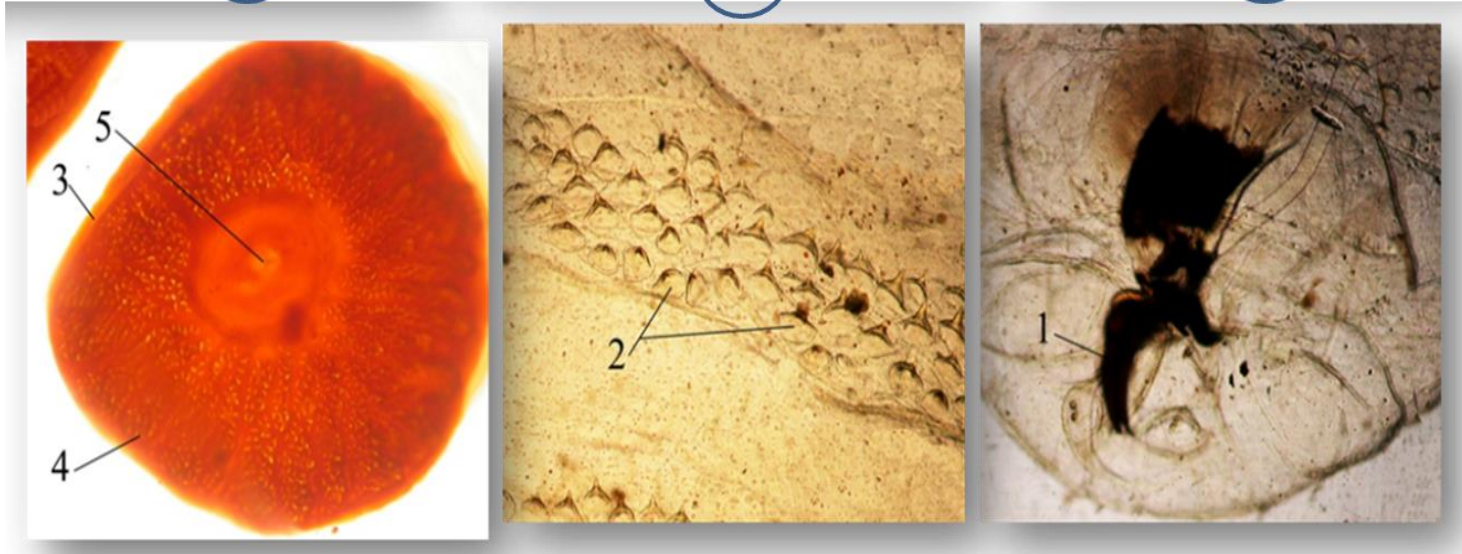

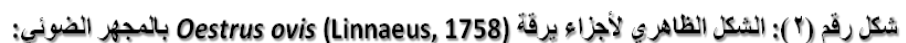

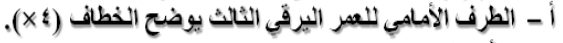

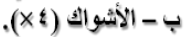

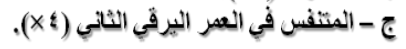
الخطاف.

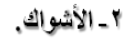

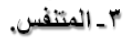

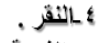

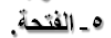




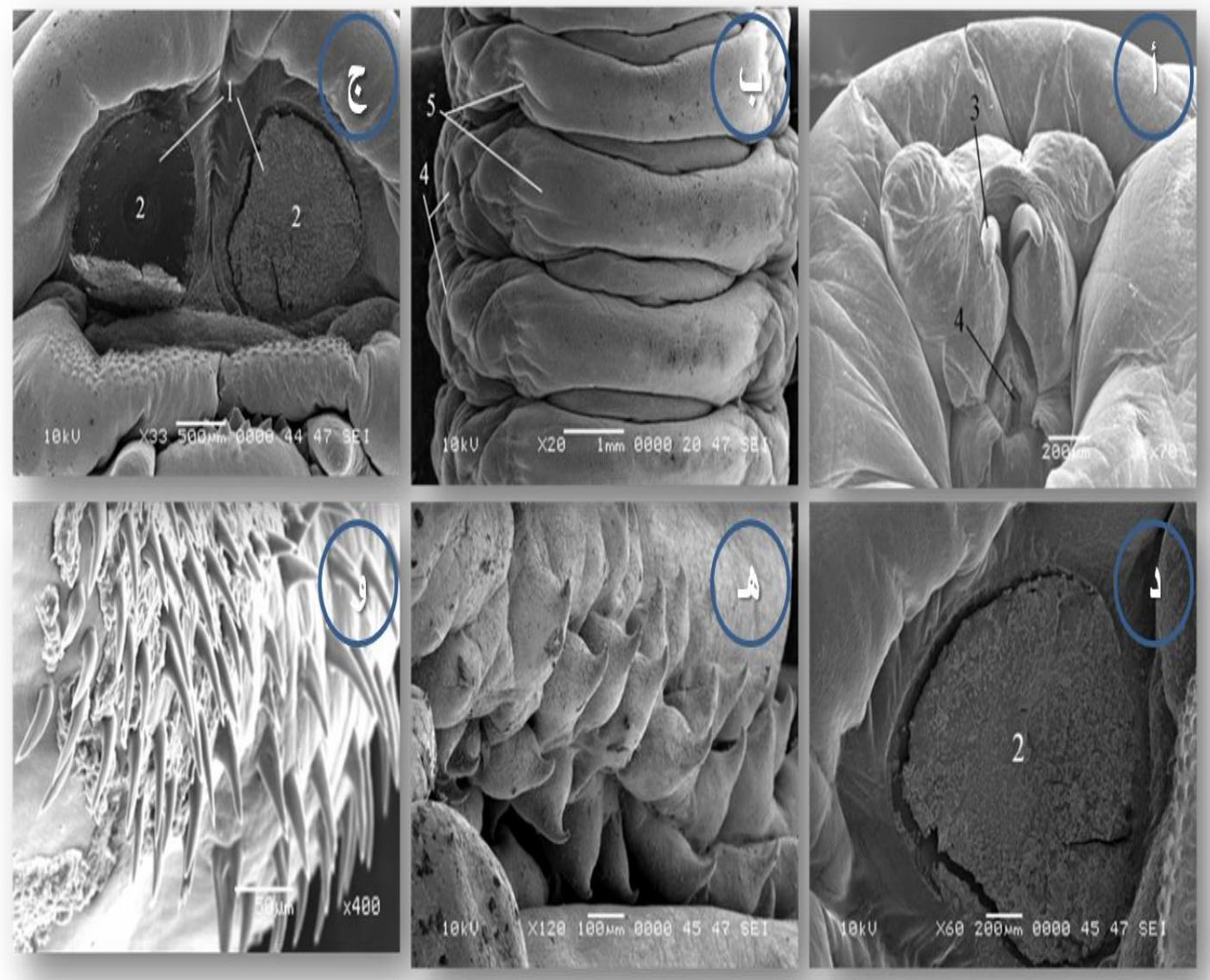

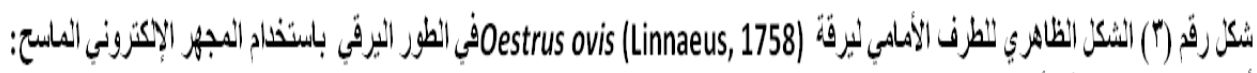

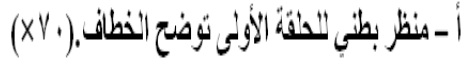

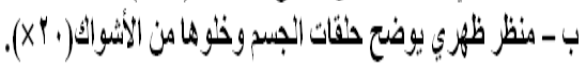

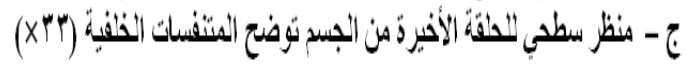

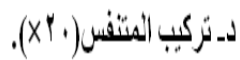

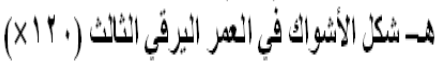

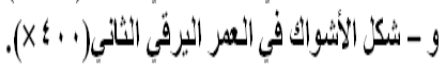

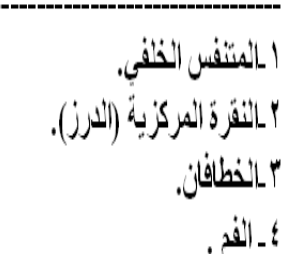


الأولى، ويتناقص إلى صفان في الثلاثة العقل الأخيرة،

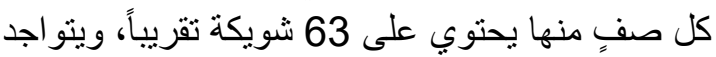

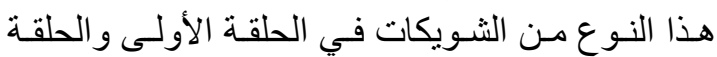
الأخيرة بكثرة عند إنغمادات الجسم ل الفيكات

الخطـاطيف الفميـة Mouth-Hooks تحتـوي الحلقـة

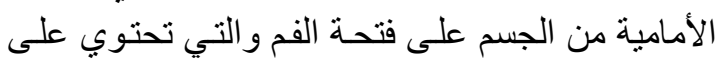

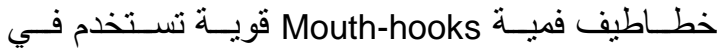

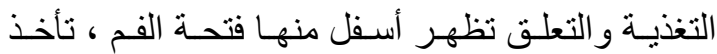

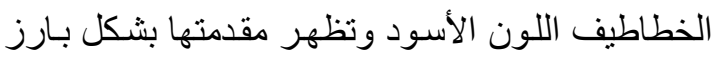
من خلال الطرف الأمامي للحلقة الأولى ، بينمـا تكون

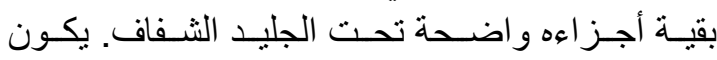

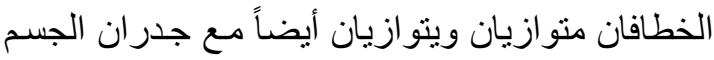
الجانبية حيث يتخذان وضع عمودي مع حلقان انبات الجسم.

الثغور التنفسية Spiracles تحتوي الحلقة الأولى من

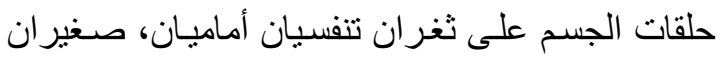
في الحجم مو ازيان للخطاطيف الفمينة، ينتهي كل منهيا بأربع حلمات مزدوجة الرأس، وتحتوي الحلقة الأخيرة

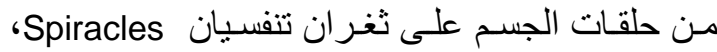

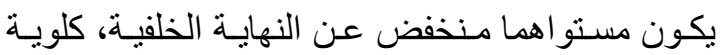

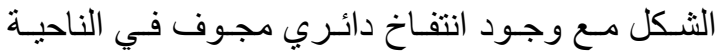
الداخلية يحتوي على الدرز أو النقرة ـ وتكون صفيحة

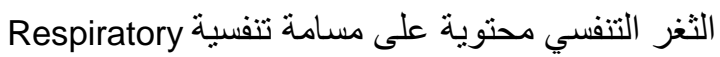
pore

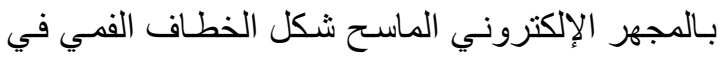
يرقات Oestrus ovis و التي تكون منحنية لأسفل غير الإني

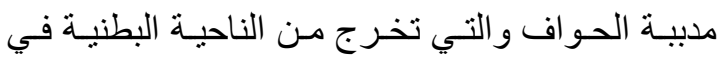

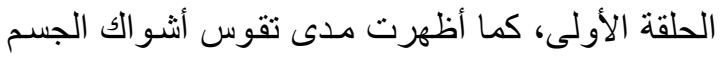

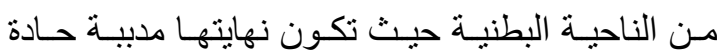

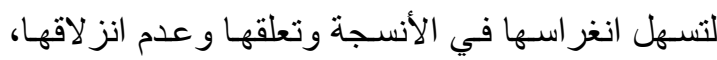

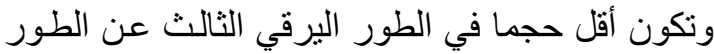

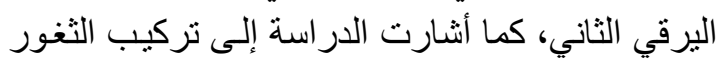

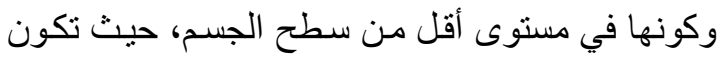

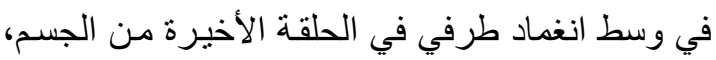

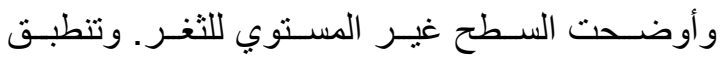

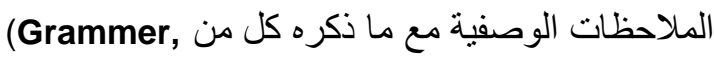
حيث تمت et al 1995)
تلدويـ أنف الجمال Cephalopina titillator (Clark 1797)

\section{وصف اليرقه Descriptionha ( أثكال رقم 4 و5}

Apodous larva دوديسة عديمسة الأرجـل نهايتهــا الخلفـــة أعـرض مـن النهايــة (vermiform)

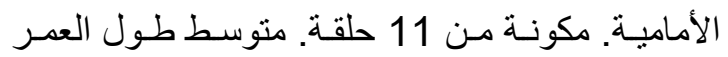

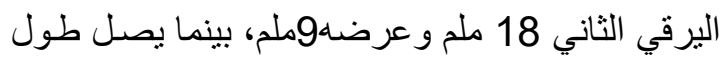
العمر اليرقي الثالث إلى 27 ملم و عرضة إلى 11 11ملم.

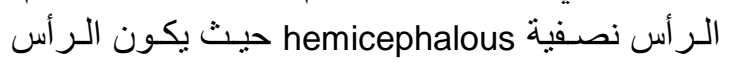

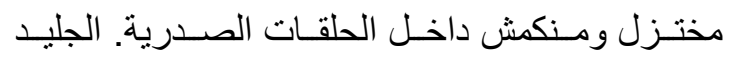
cuticle بقع بنية في السطح الظهري عند النهاية الخلفية ، بينما الطور الثالث لا يحتوي على أي بقع لونيـة ـ. الجليد

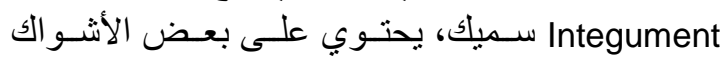
Spine

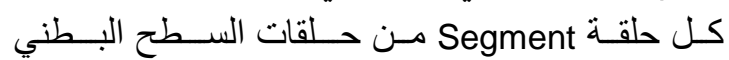

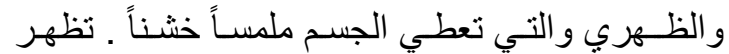

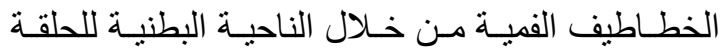
الأولى من حلقات الجسم، يحيط بها الثخر ان التهان التنفسيان

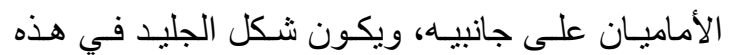

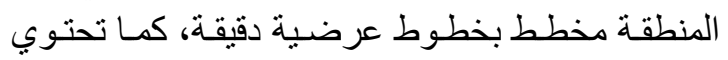

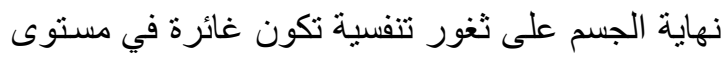

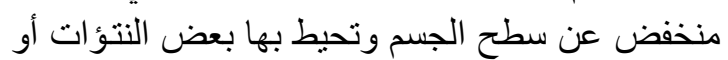
الحلمات الحسية و التي تكون أطول من الناحية البطنيـة

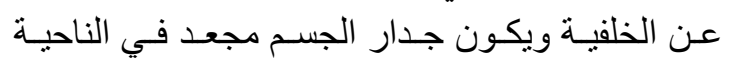

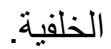

توزيع الأشواك Spines Distribution هناك نوعان

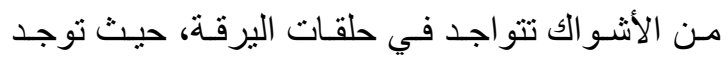

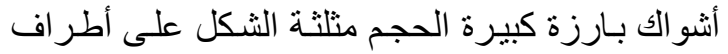

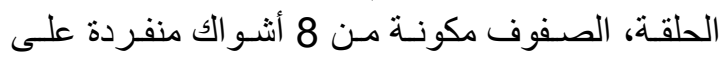

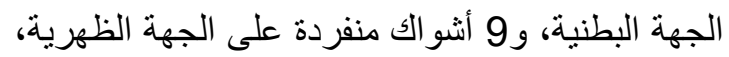

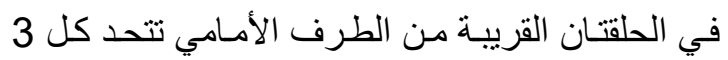

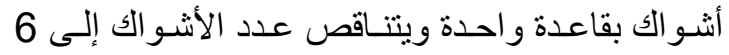

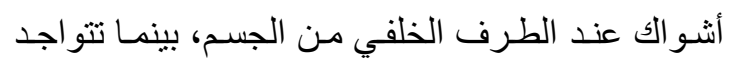

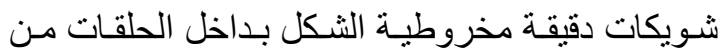
الناحيـة البطنيـة فقط، تترتب في 3 صفوف في العقل 


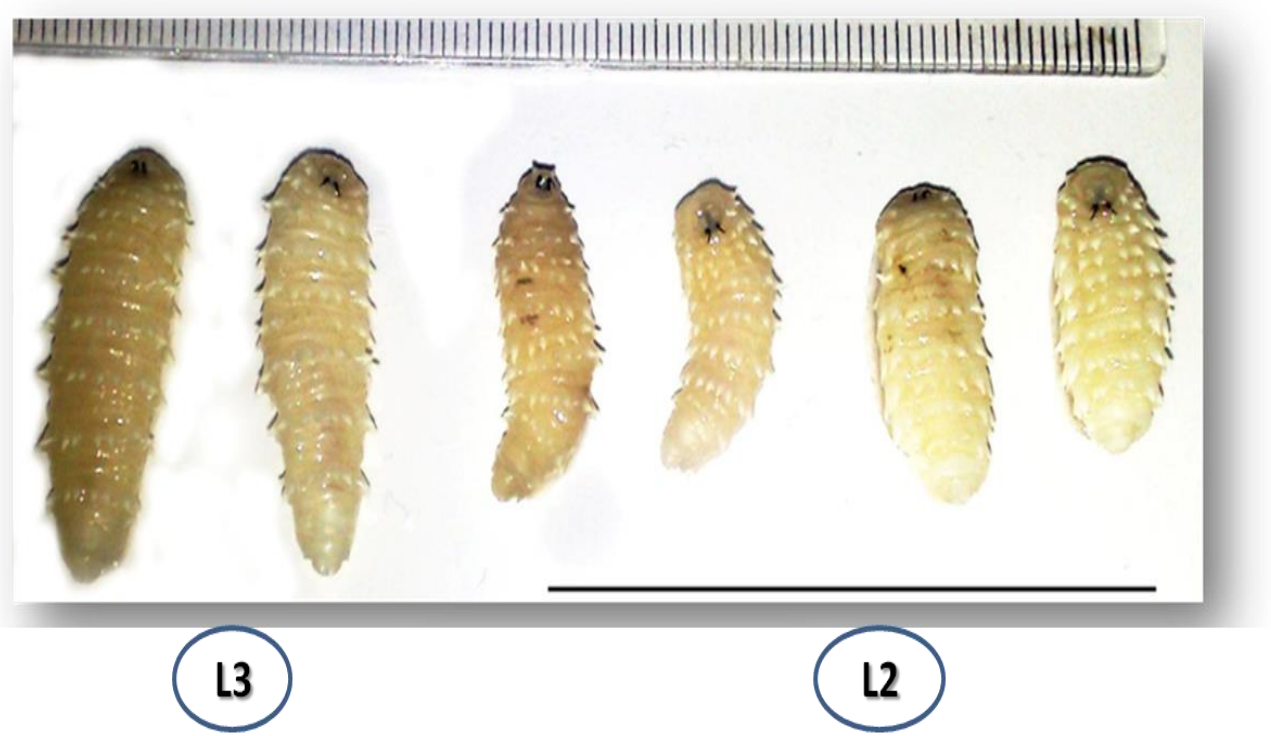

\section{شكل رقم (9): الثكل الظطاهري لأطوار يرقة (Clarck, 1797)}
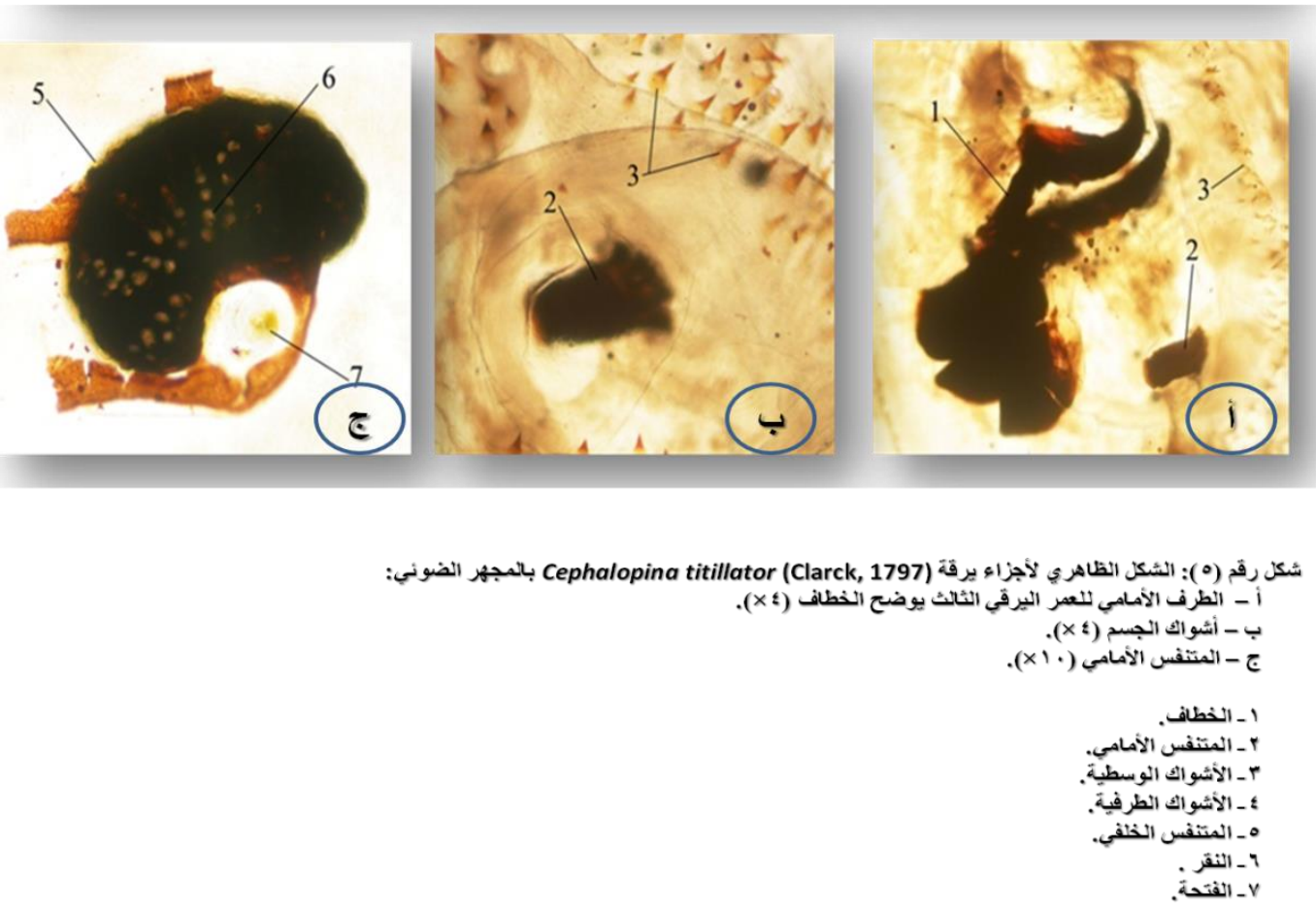


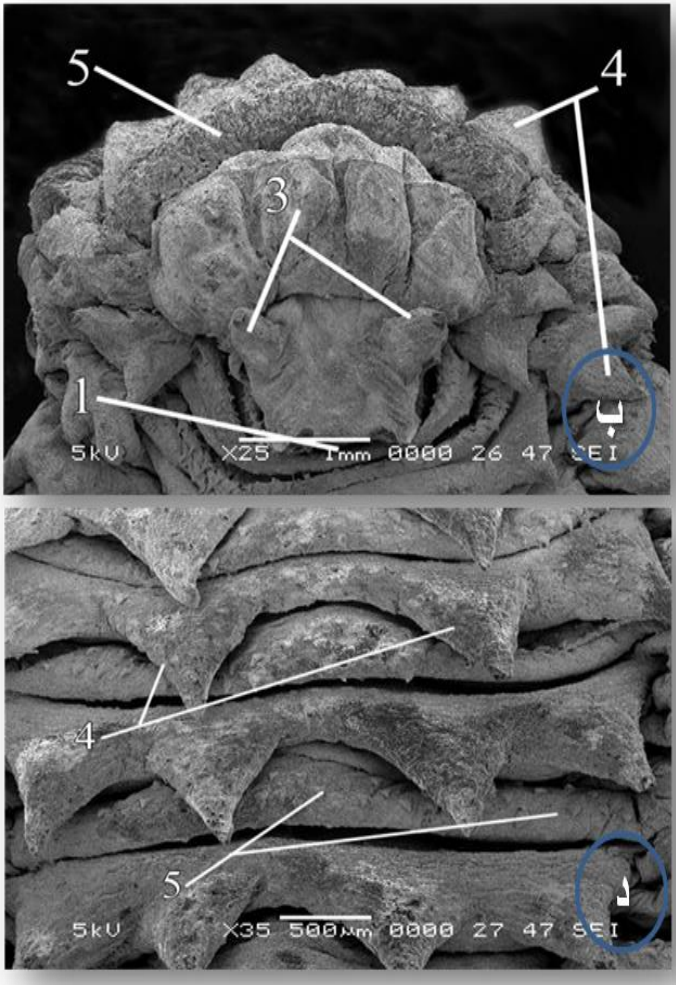

هـ أسُوا الك الجسم الوسطبة. 1- جلار الجسب المخطط.

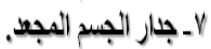

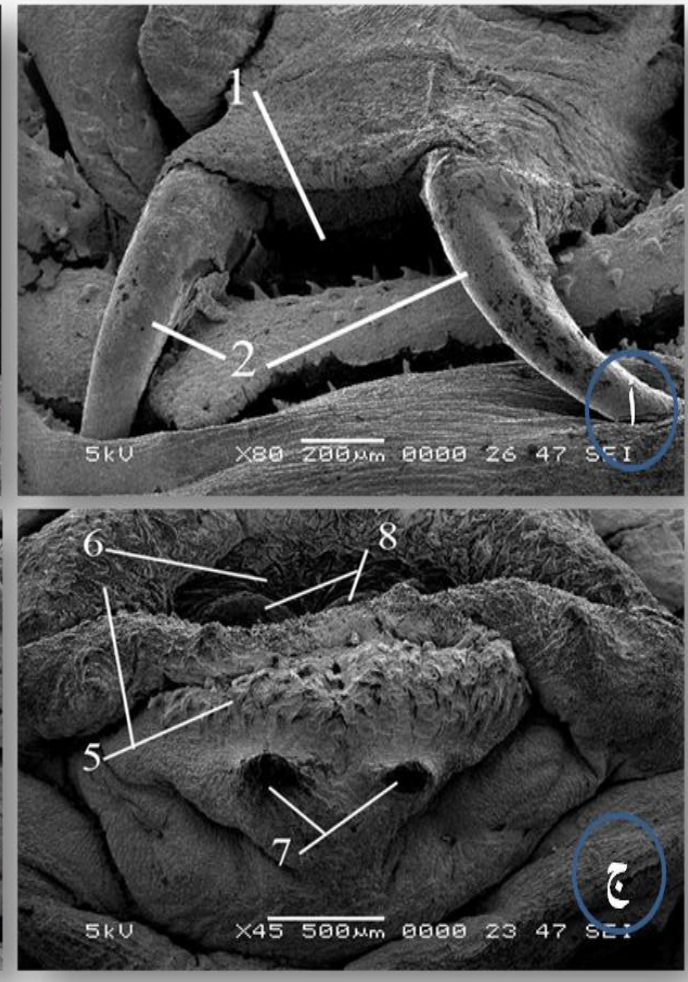

Cephalopina titillator (المجهر الإكتروني المانيح: (Clarck, 1797)

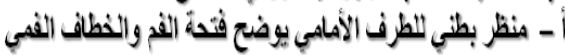

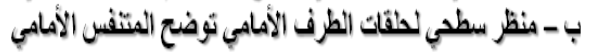

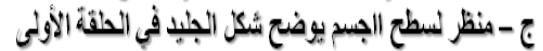
لدـ منظر بطني لسطح الجسم يوضح شئكل الجليد في الحلقة الخلفية

$$
\begin{aligned}
& \text { ا ـ فتحة الفح. } \\
& \text { بالخطاف الفي الخي }
\end{aligned}
$$

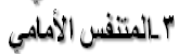

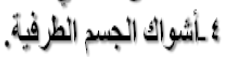

الدر اسـه بـالمجهر الضـوئي، (Stevens, et al 1991) و (Guitton, et al 2001) و (Gaqabi, 2005) (G) (G) و (Giannetto, et al 1999) حيـث تمـت الدراسـة (Guitton)

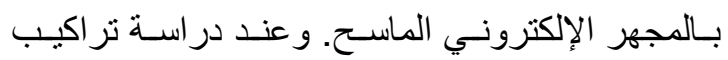

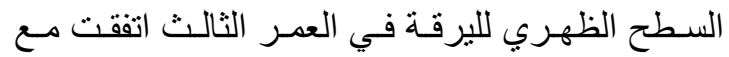

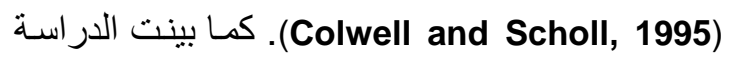

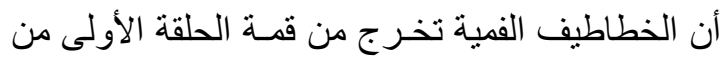

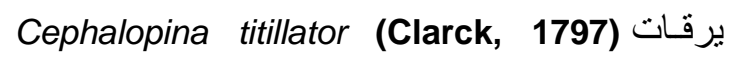
وتكـون منحنيـة ( متجهـه) إلى أسـفل، كمـا أن سـطح 
Arabia. Bull. Ent. Sco. Egypt, 82: 2133.

Beccari, F. (1971). Contributo Alla Conoscenza \& Entomofauna Dell. Arabia Saudita. Riv. Agric. Subtrop. E trop. 65:21c.

Bland, R.G. and H.E. Jaques (1980). How to Know the Insects. The Picture Key Nature Series. W.M.C. (3th Ed.) p. 315. Brown Company Publishers Dubuque, lowa New York.

Borror, D.J.; C.A. Triplehorn and N.F. Johnson (1989). An Introduction to the Study of Insects (6th Ed.). Ch32. p. 499. Saunders College Publishing, London.

Brisou, P. and G. Menard (2000). External ophthalmomyiasis by Oesterus ovis from a beach in Var. Med. Trop. (Mars). 60: 64-70.

Buttiker, W. and F. Zumpet (1982). Vetrinary and Applied Zoology in Saudi Arabia Myiasis in Domestic Animals. Funa of Saudi Arabia, 4: 520-524.

Colwell, D.D. and P.J. Scholl (1995). Cuticular sensilla on newly hatched larvae of Gasterophilus intestinalis and Oestrus ovis. Med. Vet. Entomol. 9: 85-93.

El-Azazy, O.M. (1990). Wound myiasis caused by Cochliomyia hominivorax in human in Libya. Trans R. Soc. Trop. Med. Hyg. 84: 747-755.

El-Azazy, O.M. (1992). Observation on the New World screwworm fly in Libya and the risk its entrance into Egypt. Vet. Parasitol. 42: 303-113.

Fekry, A.A.; A.O. El-Serougi and S.A. Ayoub (1997). Oestrus ovis (sheep nasal fly) infesting the eyes and the nose of a camel keeper family. J. Egypt. Soc. Parasitol. 27: 493-499.

Giannetto, S.; V. Santoro and S. Pampiglione (1999). Scanning microscopy of Osterus ovis larva (Diptera:Oestridae) Skin armour and posterior spricles, Parasitol. 6: 73-80.

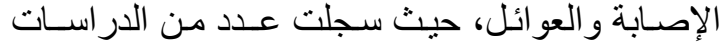

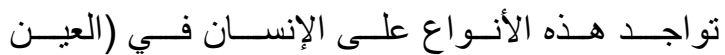

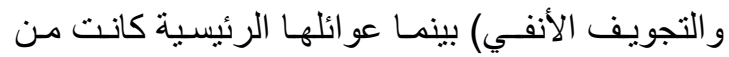

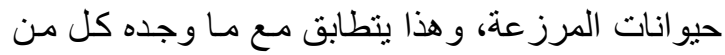

'(Wolfeschneider and Wiedemann, 1996)

‘(Fekry, et al 1997) ، (Lucientes, et al 1997)

(Brisou and ، (Victor and Bhargva, 1998)

(Suzzoni-Blatger, et al ، Menard, 2000)

(El- ، (Prosl and Meyer, 2003) 9 2000)

(Saraiva, و (El-Azazy, 1992) ‘Azazy, 1990)

.et al 2006) الثكر والتقدير

\section{ACKNOWLEDGEMENTS}

الشكر والتقدير لمدينة الملك عبد العزيز للعلـوم

والتتقية بالمملكة العربية السعودية التي قامت بتمويل وايل

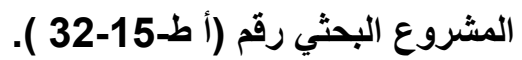

المراجع REFERENCES

$$
\text { أولاً: المراجع العربية }
$$

دبــور، علــي ابـرا هيم وموســى، محمـــ الضــوي

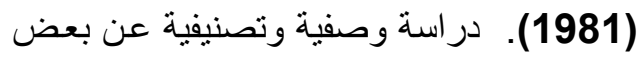

أنواع الذباب في المملكة العربية السعودية. نشرة

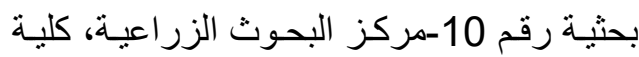

الزر اعة، جامعة الملك سعود، الرياض.

ثانياً: المراجع الأجنبية

Al-Behbehani, B. (2006). SEM of the Oestrus ovis larvae causing myiasis of sheep in the state of Kuwait with a review on human infection. J. Egypt. Soc. Parasitol. 36: 643-697.

Al-Saqabi, S.M. (2005). Scanning electron microscopy Diptera:Ostridae of Osterus ovis larvae recovered from sheep coetaneous myiasis in Saudi 
Grammer, J.; C. Erb; G. Kamin; M. Wild; C. Riedinger; P. Kosmidis; U. Pleyer and H.J. Thiel (1995). Ophthalmomyiasis externa due to the sheep botfly Oestrus ovis (Diptera: Oestridae) in southwest Germany. Ger. J. Ophthalmol. 4: 188-283.

Guitton, C.; J.M. Perez and P. Dorchies (2001). Scanning electron microscopy of larval instars and imago of Oestrus caucasicus (Grunin, 1948) (Diptera: Oestridae). Parasite. 8: 155-215.

Hussein, M.F.; F.M.; Elamin; N.T. ElTaib and S.M. Basmaeil (1981). Pathology to Nasopharyngeal Myiasis in Saudi Camels (Camelus dromedarius). Saudi Biol. Soc. $5^{\text {th }}$. Symposium, p. 67.

Innocenti, L.; P. Lucchesi and F. Giorgi (1997). Integument ultrastructure of Oestrus ovis (L.) (Diptera: Oestridae) larvae: host immune response to various cuticular components. Int. J. Parasitol. 27: 495-506.

Keirans, J.E.; C.M. Clifford and D. Corwin (1976). Ixodes sigelos n. sp. (Acarina: Ixodidae), a parasite of rodents in Chile, with a method for preparing ticks for examination by scanning electron microscopy. Acarologia. 18: 217-225.

Lucientes, J.; V. Clavel; M. FerrerDufol; H. Valles; M.A. Peribanez; M.J. Gracia-Salinas and J.A. CastiIlo (1997). Short report: one case of nasal human myiasis caused by third stage instar larvae of Oestrus ovis. Am. J. Trop Med Hyg. 56: 608-617.

Omar, M.S.; A.B. Das and N.I. Osman (1988). External ophthalmomyiasis due to the sheep nostril botfly larva Oestrus ovis in Saudi Arabia. Ann. Trop. Med. Parasitol. 82: 221-224.

Pritchard, M.H. and G.O.W. Kruse (1982). The Collection and Preservation of Animal Parasites, 141 pp. University of Nebraska Press, Lincoln and London.
Prosl, H. and J. Meyer (2003). Ophthalmomyiasis caused by Oestrus ovis in a 3 year old boy. Wien Klin Wochenschr. 3: 76-85.

Saraiva Vda, S.; M.H. Amaro ; R.Belfort and M.N. Burnier (2006): A case of anterior internal ophthalmomyiasis: case report. Arq. Bras. Oftalmol. 69: 741-744.

Smith, G.V. (1973). Insect and other Arthropods of Medical Importance, p. 389. The Trustes of the British Museum (Natural History). London.

Stevens, J.D.; A.C. McCartney and R. Howes (1991). Oestrus ovis ophthalmomyiasis acquired in the UK: case report and scanning electron microscopic study. Br. J. Ophthalmol. 75: 702-705.

Suzzoni-Blatger, J.; L. Villeneuve; B. Morassin and J. Chevallier (2000). A case of external human ophthalmomyiasis by Oestrus ovis in Toulouse (France). J. Fr. Ophtalmol. 23: 10201022.

Victor, R. and K. Bhargva (1998). Ophthalmomyiasis in Oman: a case report and comments. Wilderness Environ Med. 9: 32-37.

Wölfelschneider, P. and P.Wiedemann (1996). External ophthalmic myiasis cause by Oestrus ovis (sheep and goat botfly). Klin Monatsbl Augenheilkd. 209: 256-264.

Zayed, A.A.; S. Abdel-Shafy and R.M. El-Khateeb (2008). Surface Ultrastructure of Posterior Abdominal Spiracles of Third instars of Nasal Bots of Cephalopina titillator, Oestrus ovis ana Rhinoestrus purpureus (Diptera: Oestridae) Infestinf Camels, Sheep and Donkeys in Egypt. Research Journal Parasitology, 3: 1-11.

Zumpt, F. (1965). Myiasis in Man and Animal in the Old World, Vol. XV, p. 267. Butterworth, London. 